\title{
Aplicación de experiencias innovadoras en el ámbito educativo a través del modelo Flipped Classroom
}

DOI: $10.46932 / \mathrm{sfjdv} 2 \mathrm{n} 1-081$

Received in: November 1st, 2020

Accepted in: December 30th, 2020

\section{Carolina Real Torres}

Profesora Titular de Filología Latina

Universidad de La Laguna.

Plaza del Rector D. José Carlos Alberto Bethencourt Apartado 456. Código postal 38200. San Cristóbal de La Laguna. S/C de Tenerife (Islas Canarias, España).

E-mail: carrel@ull.edu.es

\section{RESUMEN}

La Universidad del siglo XXI requiere un abordaje del proceso de enseñanza-aprendizaje desde nuevas perspectivas que preparen a los futuros profesionales para afrontar los retos que la sociedad actual demanda. En este sentido, planteamos como experiencia educativa novedosa la aplicación del modelo Flipped Classroom a la enseñanza de la cultura clásica, resaltando la importancia de la elaboración de materiales didácticos multimedia interesantes y motivadores para el alumnado, apoyados en el uso de las nuevas tecnologías, con el objetivo de mejorar la praxis docente mediante la organización del trabajo en el aula de forma activa y participativa.

Palabras clave: Flipped Classroom, Cultura clásica, Innovación educativa, TIC.

\section{INTRODUCCIÓN}

Es una realidad el hecho de que hoy en día el sistema educativo se enfrenta a numerosos retos debido al auge de las nuevas tecnologías de la información y la comunicación (NTICs), que han cambiado radicalmente en la docencia la forma tradicional de enseñar y transmitir los conocimientos. Los nuevos tiempos nos traen también nuevos alumnos y alumnas con distintas necesidades educativas, a la vez que nos abren muchas posibilidades en nuestro entorno, como son la búsqueda de información, la elaboración y publicación de trabajos, o la comunicación entre estudiosos de un mismo campo.

Actualmente disponemos de numerosas estrategias para estimular el esfuerzo del estudiante y promover su capacidad para aprender por sí mismo y con otros, y todo ello gracias al proceso de alfabetización digital que experimentamos desde hace varios años. Las NTICs se han convertido en un gran instrumento para la difusión de información de todo tipo, mejorando las posibilidades de integrar la comunicación verbal y visual, y haciendo posible la aparición de nuevos enfoques pedagógicos referentes a los contenidos del currículo, el rol del profesorado y los nuevos entornos de aprendizaje que se ofrecen al alumnado (Cabero y Barroso, 2013). 
Las nuevas tecnologías también han llegado al entorno de los “clásicos”. Los docentes disponemos de numerosos recursos online para abordar la enseñanza de la lengua y la cultura clásica en general de una forma innovadora. En este contexto, presentamos la aplicación de Flipped Classroom (FC) a la materia de Cultura Clásica. Se trata de un modelo pedagógico que plantea la necesidad de transferir parte del proceso de enseñanza y aprendizaje fuera del aula con el fin de utilizar el tiempo de clase para el desarrollo de procesos cognitivos de mayor complejidad. Este modelo fomenta un estilo de aprendizaje dinámico en el que el estudiante tiene un mayor protagonismo, ya que previamente se ponen a su disposición los contenidos, para que en la sesión presencial se utilicen estrategias de asimilación mediante el trabajo colaborativo y cooperativo (Galindo y Badilla, 2016).

El éxito de este sistema viene avalado por el hecho de que los estudiantes son más activos en clase, el profesorado está más motivado y, en algunos casos, los alumnos y alumnas obtienen mejores resultados en los exámenes. Al desarrollarse en un ambiente interactivo donde el profesor guía a los estudiantes, éstos asumen de manera activa la construcción de su propio conocimiento mediante la búsqueda, selección y síntesis de la información, desarrollando competencias de comunicación, indagación, pensamiento crítico y resolución de problemas, entre otras (Turón, Santiago y Díez, 2014).

Los cuatro pilares sobre los que se apoya este modelo permite al profesorado

crear espacios adaptables donde el alumnado elige cuándo y dónde aprende, siendo flexibles en sus expectativas, en los tiempos de aprendizaje y en la evaluación (flexible environment); los docentes maximizan el tiempo de clase, seleccionando cuidadosamente la información y actividades correspondientes, con el fin de adoptar métodos y estrategias activas de aprendizaje centrados en el alumnado (intentional content), observan continuamente a sus estudiantes, proporcionándoles retroalimentación relevante en cada momento, así como una evaluación de su trabajo (professional educator), y les permiten participar activamente en la construcción del conocimiento, al tiempo que evalúan su aprendizaje de una manera significativa (learning culture).

En definitiva, los estudiantes trabajan los conceptos por sí mismos, usando normalmente vídeos educativos que han sido previamente preparados por sus docentes o bien cualquier otro material multimedia. En clase, en cambio, el plan es dedicar el tiempo para la discusión, resolución de dudas y tareas más creativas que requieran la presencia y el asesoramiento del profesorado. Esto puede llevarse a cabo en grupos, o bien individualmente, lo que permite marcar diferentes ritmos para cada estudiante según sus capacidades y, además, mejorar el ambiente de trabajo en el aula gracias al rol activo de cada uno (Martínez y Esquivel, 2014; Perdomo Rodríguez, 2016). 
Así, el aula invertida modifica la clase tradicional basándose en el hecho de que el alumnado identifica contenidos disciplinares a través de soportes tecnológicos utilizados fuera del aula, desarrollando un aprendizaje autónomo, mientras que los docentes se transforman en investigadores, generadores de contenido y mediadores del aprendizaje (Ouda, 2016). Por todo ello, con el fin de mejorar la calidad del aprendizaje, tendremos en cuenta las NTICs, las cuales, trasladadas al ámbito educativo y utilizadas de manera apropiada, pueden ser un extraordinario recurso tanto para el profesorado como para el alumnado, ya que ofrecen un medio eficaz, atractivo y fácilmente accesible para enriquecer el currículo y las actividades desarrolladas en el aula.

Asimismo, somos conscientes de la importancia de la integración de materiales multimedia, que se ha convertido en una práctica habitual en la educación universitaria, debido a que están en consonancia con la manera de aprender del ser humano dentro del nuevo modelo de sociedad, en la cual la revolución tecnológica tiene cada vez una mayor incidencia (Dede, 2000; Peters, 2001). Igualmente, el uso de dispositivos móviles ha demostrado ser una herramienta eficaz en relación a sus posibilidades educativas, ya que es un instrumento que pertenece al día a día de los estudiantes en su vertiente más lúdica, y la utilización de los mismos es percibida como algo positivo e interesante en su formación (Moreno, Leiva y Matas, 2016).

\section{METODOLOGÍA}

Siguiendo el modelo FC, nuestra propuesta metodológica se divide en tres fases. En la primera, tras seleccionar y clasificar los contenidos, hemos procedido a grabar un vídeo con Camtasia, programa específico para crear presentaciones vía screencast (Silva, 2012), y, posteriormente, lo hemos editado convirtiéndolo en un videoquiz, con el propósito de compartirlo con nuestros estudiantes, para que pudieran visualizarlo en casa y realizar las actividades en él propuestas antes de la primera sesión de clase. Además de las ventajas que supone el uso del vídeo, en tanto que facilita la adquisición de conocimientos al presentar el contenido de forma más amena, pues la combinación de imagen y sonido nunca falla, evitando al profesorado repetir las explicaciones y pudiendo dedicar el tiempo en el aula a otras actividades más productivas, y en tanto que ayuda al estudiante que no pudo asistir a clase ese día, se adapta perfectamente a cualquier dispositivo móvil, y tiene una mayor difusión, ya que puede compartirse fácilmente en las redes sociales, añadimos la potencialidad didáctica del videoquiz, ya que al realizar preguntas de forma progresiva e interactiva a tiempo real, se consigue que aumente el nivel de atención del alumnado (Lacher, 2015).

En una segunda fase, realizamos un debate y puesta en común en el aula, durante la cual los estudiantes, divididos en grupos, intercambiaron opiniones sobre el contenido del vídeo y resolvimos las 
dudas que se fueron planteando. Como complemento a la sesión presencial se realizó un cuestionario online para comprobar el nivel de comprensión de los contenidos.

En una tercera fase, recomendamos actividades que ayudaran a consolidar los conceptos adquiridos en forma de tareas complementarias, lo que nos brindó la posibilidad de hacer hincapié en los estudiantes más atrasados y ofrecer nuevas oportunidades a los más aventajados.

Para evitar caer en la rutina, modificamos el espacio inmobiliario y creamos secuencias dinámicas de cambio de grupos, ya que cambiar la disposición de los pupitres cambia la dinámica de las clases y fomenta el desarrollo de habilidades sociales y la inteligencia emocional. En cuanto al material, para evitar la posible carencia de un ordenador portátil en el aula, propusimos varias alternativas, como el uso de Tablet o Smartphone.

Por último, apostamos por una evaluación formativa que contemplara los esfuerzos individuales de los miembros del equipo (autoevaluaciones individuales) y el producto final producido por el grupo.

\section{PROYECTO}

Bajo el lema "los seres fabulosos de la mitología clásica", ofrecemos una serie de actividades de aprendizaje interactivas que tienen como objetivo que el alumnado haga un buen uso del tiempo asignado, investigando, seleccionando y analizando información para mejorar su comprensión sobre un tema en concreto. Constituyen estrategias útiles para adquirir información y practicar habilidades y procedimientos relacionados con las NTICs, a la vez que nos permiten valorar todo el proceso de aprendizaje. El tema que hemos elegido resulta, por su naturaleza, altamente motivador para el alumnado y puede adaptarse a cualquier nivel educativo, desde Secundaria, Bachillerato o Universidad.

Esta experiencia formativa se ha llevado a cabo con un grupo de estudiantes del Grado en Estudios Clásicos de la Universidad de La Laguna. Los objetivos concretos que se persiguen son:

- Incrementar la motivación del estudiante mediante la introducción de actividades interactivas que faciliten el estudio de los contenidos a tratar.

- Ampliar los contenidos y conocimientos que se adquieren en el aula.

- Valorar la importancia de las nuevas tecnologías.

- Utilizar recursos didácticos multimedia sobre cultura clásica para mejorar el proceso de enseñanza-aprendizaje, estimular la participación activa y el auto-aprendizaje del alumnado.

- Mejorar la comunicación y la interacción del profesor con los estudiantes y de los estudiantes entre sí. 
El vídeo (https://www.youtube.com/watch?v=LhiIKfs-sok) incluye una descripción y un comentario de las características de los cíclopes según los testimonios escritos de la literatura grecolatina, tratando la importancia que el mito original tuvo en la edad antigua y su influencia en las creencias populares y en la literatura occidental. La evolución del mito desde su orígenes y las distintas interpretaciones sobre la existencia de los cíclopes gira en torno a la tesis de Othenio Abel, paleontólogo de la Universidad de Viena, quien propuso en 1914 que dicho mito se origina de la observación y mala interpretación de restos fósiles de cráneos de mamuts enanos, como el Elephas falconeri, especie de 90 cm de altura con un cráneo un poco más grande que el de un ser humano. Sin sus colmillos, el cráneo de un mamut enano es aún más parecido al de un ser humano, por lo que los antiguos griegos, que no conocían a los elefantes, al contemplar el cráneo sin colmillos, seguramente pensaron en un extraño ser de un único ojo, de aspecto humanoide y más grande de lo normal. Así se origina el mito de una raza de gigantes de un solo ojo que habitaba en grutas en las islas del Mediterráneo, tal como relatan los testimonios escritos del s.VIII a.C. Finalmente, como ejemplo de la pervivencia del mito en la actualidad, la escultura "El Cíclope" de Jean Tinguely, pintor y escultor suizo famoso por sus "máquinas esculturas" o arte cinético, se hace eco de la imagen ovidiana de Atis transformado en río, tras morir a manos de Polifemo, el cíclope más famoso de todos los tiempos, demostrando que los seres fabulosos tienen aún cabida en nuestra imaginación.

Tras editarlo en formato videoquiz, lo alojamos tanto en nuestro blog personal (https://humanidades.blog/2017/02/28/videoquiz/), como en Educaplay (https://es.educaplay.com/es/recursoseducativos/2839309/seres_mitologicos_ciclopes.htm), una plataforma gratuita que nos ofrece estadísticas para evaluar los resultados de aprendizaje y a la que el alumnado puede acceder tras previo registro en la misma.

En las sesiones presenciales, después de resolver las dudas surgidas de la realización del videoquiz y tras un breve debate en el que se plantearon algunas cuestiones sobre la veracidad del mito y sobre las fuentes literarias, nos dispusimos a evaluar los conocimientos adquiridos mediante un cuestionario, dividiendo a la clase en grupos. En este caso, optamos por una herramienta gratuita como Kahoot!, que permite la creación de cuestionarios online donde los propios estudiantes son los concursantes.

El Kahoot!, creado con las preguntas realizadas por ellos mismos en clase, permite que cada uno pueda conocer su puntuación, estableciéndose un ranking de respuestas. La aplicación permite exportar los resultados a Excel o incluirlos en Google Drive, para que el profesorado pueda disponer de los mismos para el proceso de evaluación (Alba, Moreno y Ruiz, 2015).

Por último, como actividad de repaso y conclusión a lo aprendido, proponemos dos tareas alternativas a las que cada grupo podía optar según sus preferencias: a) buscar alguna obra literaria de 
cualquier género y época que tratara el mito de los cíclopes y elegir un breve fragmento que describiera sus características, o b) crear un relato sobre estos seres fabulosos, que podía ser enriquecido con ilustraciones propias. Estas actividades dan al alumnado autonomía, ofreciéndole opciones, ofreciéndoles fuentes de información variadas y fomentando el uso de la imaginación, la exploración y la experimentación. El resultado de dichas tareas fue plasmado en forma de Popplet, una herramienta online para crear mapas mentales o murales digitales con grandes posibilidades gráficas y de gran atractivo visual, que permite a nuestros estudiantes colgar, organizar y relacionar ideas, pudiendo añadir imágenes, textos, dibujos y vídeos de forma muy sencilla, y cuyo resultado final puede compartirse en la red o descargarse en modo pdf, jpg o png. A la hora de evaluar las aportaciones individuales dentro de cada grupo no hay problema, ya que cada popplet aparece con el nombre de su autor, pues, cuando el docente pincha en "share" en el nombre de cada una de las personas que participan en el proyecto, sus popplets quedan resaltados, pudiendo evaluar tanto el trabajo individual como el resultado final del grupo.

\section{RESULTADOS}

El uso de la tecnología en cualquier entorno de aprendizaje nos ofrece la posibilidad de una atención hacia el alumnado más individualizada, un ambiente en el aula con más posibilidades para la interacción y, por consiguiente, estudiantes más motivados por aprender. En este sentido, la sencillez en el manejo de las herramientas tecnológicas empleadas (Videoquiz, Kahoot y Popplet), y el descubrimiento de sus posibilidades didácticas, han facilitado la participación del alumnado y su implicación en el proceso de aprendizaje.

El nivel de asistencia y participación de los estudiantes puede considerarse

sobresaliente (100\%), lo que, sin duda, obedece a una gran dosis de motivación. La mayoría agradeció el conocimiento y uso de este tipo de recursos por las posibilidades que puede aportarles en su futura práctica docente, de manera que los resultados fueron muy satisfactorios.

En particular, el uso de representaciones gráficas ayudó al alumnado a sistematizar, analizar, visualizar y redactar las características más importantes, centrando su atención no sólo en la definición, sino en lo más significativo, y, por tanto, lo más difícil de olvidar. En esta experiencia con Popplet el alumnado ha diseñado presentaciones con un alto impacto visual, utilizando todo tipo de recursos, especialmente los audiovisuales.

Por otro lado, el uso de dispositivos móviles a la hora de realizar el Kahoot! supuso una novedad en el ambiente universitario. Asimismo, las secuencias dinámicas de cambio de grupos y la alteración del mobiliario aportaron dinamismo a la clase y favorecieron el espíritu de equipo y el trabajo 
colaborativo.

Así pues, mediante estas herramientas y el método FC, hemos mejorado el aprendizaje que en tantas ocasiones se centra en una memorización exclusiva, y no tanto en una práctica y uso compartido.

\section{CONCLUSIONES}

La clase inversa es un modelo en el que juegan un papel esencial las herramientas TIC en tanto que nos proporcionan un análisis de las interacciones de los estudiantes, lo que nos ayuda a valorar el proceso de aprendizaje y de participación del alumnado paso a paso, complementando la evaluación del resultado final que puede proporcionarnos una rúbrica. De esta manera, todo el proceso de enseñanzaaprendizaje es cuantificable y evaluable.

Desde luego, este método resulta más efectivo porque el profesorado se adapta

a los diferentes ritmos de aprendizaje y ninguno de los estudiantes se queda atrás, asumiendo desde un principio que no todos los alumnos y alumnas van a avanzar a la misma velocidad, y mejorando notablemente la relación profesor-alumno (Bishop y Verleger, 2013). En efecto, cuando el profesorado no está de pie frente a sus estudiantes simplemente hablando y puede circular por la clase, la relación cambia por completo (Del Valle, 2021: 68).

En definitiva, los resultados de esta experiencia muestran que los estudiantes se sienten más comprometidos con su propio aprendizaje. Y lo que es más importante, valores fundamentales como el espíritu de equipo, la cohesión y la motivación se ven fortalecidos, pues la Educación Superior no sólo tiene la responsabilidad de formar buenos profesionales, sino también debe hacer un esfuerzo por promover valores como la solidaridad y la responsabilidad (Folgueiras, Luna y Puig, 2013), siendo el marco ideal para potenciar el compromiso cívico-social del estudiantado y valores de cooperación, respeto y empatía.

Como conclusión, vemos que el aula ya no es el único lugar de aprendizaje y que, con el avance imparable de Internet como fuente de información, es posible adquirir por medio de estrategias pedagógicas conocimiento a través de la red. Recordemos también que el FC pretende, ante todo, aumentar la eficiencia del tiempo de clase y mejorar la comprensión del estudiante, por lo que este modelo puede servir para cualquier materia y cualquier nivel de estudio, independientemente de la edad del alumnado. Estamos ante la juventud de la era tecnológica, y aplicar estas herramientas y apostar por medios y recursos audiovisuales es hacerlo, sin lugar a dudas, por la mejora docente. 


\section{BIBLIOGRAFÍA}

Alba Ferré, E., Moreno, Blesa, L., Ruiz González, M. (2015). The Star System Apps to Bridge Educational Gaps: Kahoot, Screencast y tableta gráfica. XII Jornadas Internacionales de Innovación Universitaria Educar para transformar: Aprendizaje experiencial. Universidad Europea de Madrid, 791799.

Bishop, J. L. y Verleger, M. A. (2013). The flipped classroom: A survey of the research. En ASEE National Conference Proceedings. Atlanta, GA, 30(9), 1-18.

Cabero, J. y Barroso, J. (2013). La escuela en la sociedad de la información. La escuela 2.0. En J. Barroso y J. Cabero (coords.). Nuevos escenarios digitales: las tecnologías de la información y la comunicación aplicadas a la formación y desarrollo curricular (21-36). Madrid: Ediciones Pirámide.

Dede, C. (2000). Emerging influences of information technology on school curriculum. Journal of Curriculum Studies, 32(2), 281-303. JOUR. http://doi.org/10.1080/002202700182763

Del Valle Godoy, S., Marucco, E. S., Negrete, C. S., \& Sposetti, A. H. (2021). Compulsory virtual education at school. An Argentine experience. South Florida Journal of Development, 2(1), 65-77.

Folgueiras, P., Luna, E. y Puig, G. (2013). Aprendizaje y servicio: estudio del grado de satisfacción de estudiantes universitarios. Revista de Educación, 362, 159-185.

Galindo, J. J. y Badilla, M. G. (2016). Innovación docente a través de la metodología Flipped Classroom: percepción de docentes y estudiantes de Educación Secundaria. Didasc@lia. Didáctica y Educación, VII(6), 153-172.

Lacher, L. L., \& Lewis, M. C. (2015, February). The effectiveness of video quizzes in a flipped class. In Proceedings of the 46th ACM Technical Symposium on Computer Science Education, 224-228.

Martínez Olvera, W. y Esquivel Gámez, I. (2014). Aula Invertida o Modelo Invertido de Aprendizaje: origen, sustento e implicaciones. En I. Esquivel Gámez (coord.) Los modelos tecnoeducativos, revolucionando el aprendizaje del siglo XXI (143-160). México: Lulu.

Moreno, N. M., Leiva, J. J. y Matas, A. (2016). Mobile learning, Gamification and Augmented Reality for the teaching and learning of languages. International Journal of Educational Research and Innovation (IJERI), 6, 16-34.

Ouda Kahdri Ahmed, H. (2016). Flipped Learning As A New Educational Paradigm: An Analytical $\begin{array}{lllll}\text { Critical Study. } \quad \text { European } & \text { Scientific }\end{array}$ http://dx.doi.org/10.19044/esj.2016.v12n10p417

Perdomo Rodríguez, W. (2016). Estudio de evidencias de aprendizaje significativo en un aula bajo el modelo flipped classroom. EDUTEC. Revista Electrónica de Tecnología Educativa, 55, 1-17.

Peters, O. (2001). Learning and Teaching in Distance Education: Pedagogical Analysis and Interpretations in an International Perspective. Education + Training, 41(8), 384-386. https://doi.org/10.1108/et.1999.41.8.384.3 
Silva, M. L. (2012). Camtasia in the classroom: Student attitudes and preferences for video commentary or Microsoft Word comments during the revision process. Computers and Composition, 29(1), 1-22.

Tourón Figueroa, J., Santiago Campión, R. y Díez, A. (2014). The Flipped Classroom: Cómo convertir la escuela en un espacio de aprendizaje. Madrid: Grupo Océano. 